Proc Indian Natn Sci Acad 81 No. 1 February 2015 Special Issue, pp. 36-39

(c) Printed in India.

DOI: $10.16943 /$ ptinsa/2015/v81i1/48048

\title{
Testing of Track Point Resolution of Gas Electron Multiplier with Pion Beam at CERN SPS $\mathrm{R} \mathrm{P} \mathrm{ADAK}^{1^{*}}, \mathrm{~S} \mathrm{CHATTOPADHYAY}^{2}, \mathrm{~S} \mathrm{DAS}^{1}, \mathrm{~A} \mathrm{~K} \mathrm{DUBEY}^{2}, \mathrm{M} \mathrm{S} \mathrm{GANTI}^{2}, \mathrm{~J} \mathrm{SAINI}^{2}$ and R SINGARAJU ${ }^{2}$ \\ ${ }^{1}$ Centre for Astro-particle Physics and Space Sciences, Bose Institute, Kolkata 700 091, India \\ ${ }^{2}$ Variable Energy Cyclotron Centre, Kolkata 700 064, India
}

(Received on 30 April 2014; Accepted on 30 August 2014)

\begin{abstract}
A muon detection system using segmented and instrumented absorber has been designed for high-energy heavy-ion collision experiments to be held at GSI, Darmstadt, Germany. The muon detector system is mounted downstream of a Silicon Tracking System. The reconstructed tracks from the STS are to be matched to the hits in the GEM detector. For reconstructing track in the GEM Detector, track point resolution is an important issue. We report her first time the track point resolution of the GEM detector.
\end{abstract}

\section{Key Words : GEM Detector; CBM; Muon Detector; Segmented Absorber; Lmvm; Track Point} Resolution

\section{Introduction}

A Muon detection system using segmented and instrumented Fe absorbers has been designed for highenergy heavy-ion experiment to be held at FAIR, GSI, Germany (CBM Collaboration, 2014). In this experiment, charmonium and low mass vector mesons will be detected via their muon decay channel. This Muon Chamber Detector (MUCH) uses 6 segmented and instrumented Fe absorbers (Dubey et al., 2013). Particles while passing through the absorber segments generate secondaries and the tracking chambers face particles at a rate of 0.3 particles $/ \mathrm{cm}^{2}$. In such a condition, the most upstream chamber will be required to operate at a rate of $3 \mathrm{MHz} / \mathrm{cm}^{2}$. Operation of CBM-MUCH at high interaction rate requires a detector with large acceptance, high granularity and high rate capability. First few detector stations at MUCH will use Gas Electron Multiplier (GEM) technology (Sauli et al., 2012). We have conducted a test experiment with proto-type detectors using triple GEM at CERN-SPS with $150 \mathrm{GeV} / \mathrm{c}$ Pion beam. In CBM experiment, tracks are reconstructed using the hits recorded in a number of Silicon Tracking Stations (STS) placed inside

\footnotetext{
*Author for Correspondence : E-mail: rpadak@jcbose.ac.in
} 
a dipole magnet. Later those tracks are projected onto muon chambers for further reconstruction required for muon detection. We report here the first measurement of the track-point resolution of the GEM detector using a self-trigger read out ASIC (Application Specific Integrated Circuit).

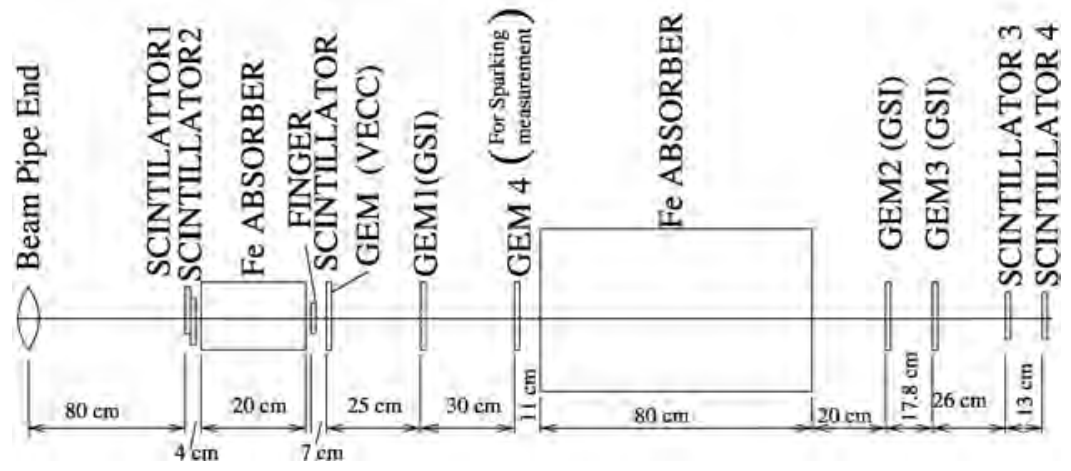

Fig. 1: Experimental Setup at CERN H4 beam line

\section{Experimental Setup}

The layout of the setup is shown in Fig. 1. In this test experiment, four prototype GEM detectors were used. One of them, is assembled at VECC and it is $10 \mathrm{~cm} \times 10 \mathrm{~cm}$ in size and consists of 512 readout pads of dimension 3mm X 3mm. The drift gap, transfer gap1, transfer gap2 and induction gap were $3 \mathrm{~mm}, 1 \mathrm{~mm}$, $1 \mathrm{~mm}, 1.5 \mathrm{~mm}$ respectively (Fig. 2) (Dubey et al., 2012) and the gas mixture used was $\mathrm{Ar}: \mathrm{CO}_{2}$ in the ratio 70:30. The resistances are so chosen that the voltage across all the GEM foils are same for the VECC GEM. The signal corresponding to a hit, is read out by four Front End Boards(FEB), each of which houses one nXYTER chip. Data acquisition and control were done by Read Out Controllers(ROC). For data acquisition two types of triggering have been used. First one is the Aux 1 trigger where 4 scintillators along with a finger scintillator has been used. In Aux2 type trigger, only 4 scintillators were used. Here in this analysis we used Aux2 as trigger.

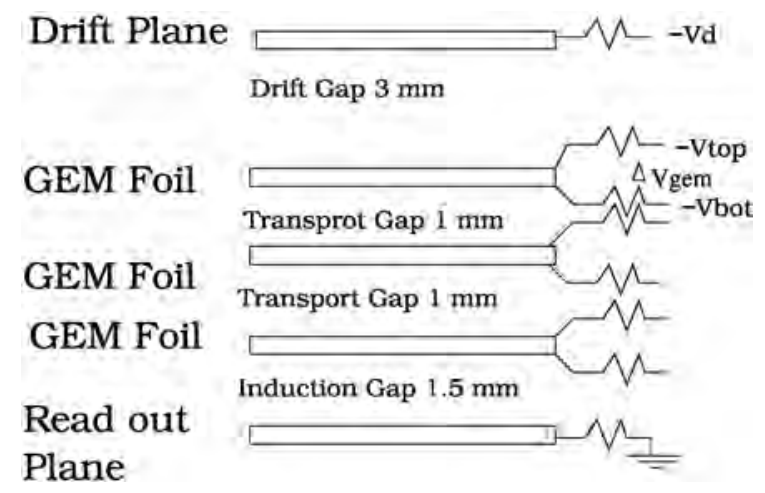

Fig. 2: Schematic layout of the triple GEM chamber 
Another three detectors were built at GSI, Germany. The drift gap, transfer gap1, transfer gap2 and induction gap of GSI GEMs were 3mm, 2mm, 2mm, 2mm respectively. Each chamber consists of 256 readout pads, each of which $6 \mathrm{~mm} \times 6 \mathrm{~mm}$ in size and readout by 2 FEBs connected to one ROC. For this self triggering system, all the hits above a predefined threshold are readout by the nXYTER. The signals are digitized and stored. Only hits with the time-correlation with the trigger window are taken for analysis.

\section{Result}

In this analysis, first the alignment of the 3 GEMs (VECC GEM, GEM1(GSI) and GEM3(GSI)) were tested. Noise in the experiment is measured by taking data in a no-beam-spill situation. The ADC values are then subtracted for each channel to obtain corrected ADC spectra. The ADC distribution is shown in Fig. 3.

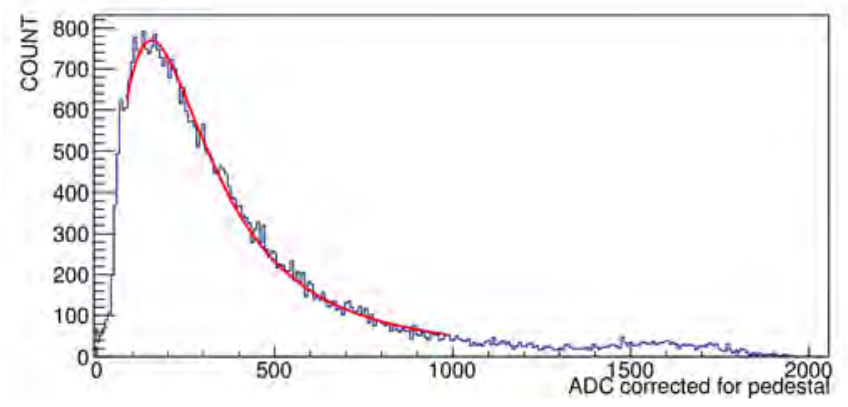

Fig. 3: ADC distribution of VECC GEM for $\mathbf{V}_{d}=\mathbf{3 0 0 0}$ Volt and $\triangle_{G E M}=334$ Volt

A readout pad with maximum ADC in an event is chosen at VECC GEM. The ADC weighted mean X, Y positions are determined from distribution of the hits from a large numbers of events (Fig. 4).

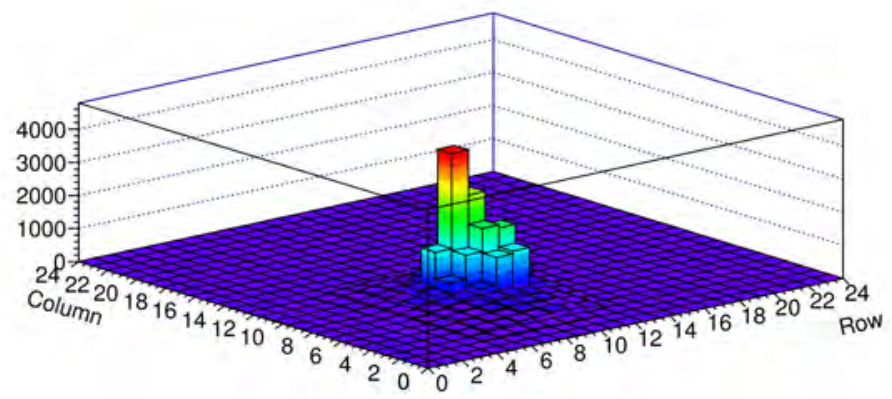

Fig. 4: Lego plot of the hits distribution for VECC GEM for $\Delta_{G E M}=334$ Volt

This mean value is subtracted from each hit in an event. This is done for all the GEMs. The events that has hit on the chosen pad at VECC GEM, GEM-1(GSI) and GEM-3(GSI) are taken for tracking. 

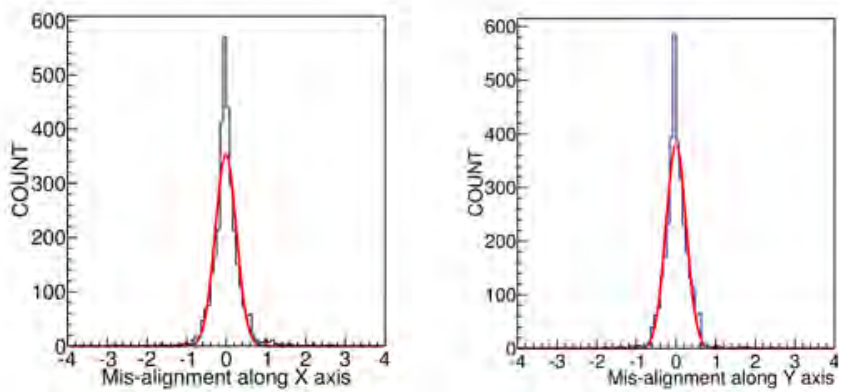

Fig. 5: Mis-alignment along $X$ and $Y$ direction

A straight line is drawn connecting the hits at VECC GEM and GEM-3. Then the corresponding x, $y$ positions are calculated from the fit. The differences between the measured position and the fitted position are plotted for all the hits. The mean of this distribution gives the mis-alignment of the GEM-1. Our result shows that the mean value of mis-alignment is negligible compared to the pad size of the GSI GEM (6mm $\mathrm{x}$ $6 \mathrm{~mm}$ ) (Fig. 5). Next, we have checked the track point resolution of the GEMs. As per Fig. 5 and considering the nominal resolution of the pads, the position resolution follows $\sigma_{G S I 1 G E M}=\sqrt{\sigma_{V E C C G E M}+\sigma_{G E M 3}}$ $\mathrm{cm}=0.19 \mathrm{~cm}$.

The extracted resolution is as per expectation from the pad size of the chamber.

\section{Acknowledgement}

We thank S K Ghosh, S Raha of Bose Institute, Kolkata, Walter Müeller, P Senger, S Linev from GSIDarmstadt, L Ropelski and E Oliveri of RD51 for all helps. We would also like to thank CERN H4 beam line crew for all possible helps. This work is supported by the DAE-SRC award under the scheme no. 2008/21/07-BRNS/2738. R P Adak like to acknowledge the University Grant Commission (UGC).

\section{References}

1. www.gsi.de/en/work/research/cbmnqm/cbm.htm

2. AK Dubey and S Chattopadhyay et al., (2013) Nucl Inst and Meth A718 418

3. F Sauli, et al., (1997) Nucl Inst and Meth A386 531

4. AK Dubey and S Chattopadhyay et al., Testing of Triple-GEM chambers for CBM Experiment at FAIR using self-triggered readout electronics (in press). 